\title{
Early Onset of Platinum Hypersensitivity in a Dentist: A Case Report
}

\author{
Maurie Markman ${ }^{a} \quad$ Daniel Lieber ${ }^{b}$ \\ aThe University of Texas MD Anderson Cancer Center, Houston, Tex., and \\ ${ }^{b}$ The Angeles Clinic and Research Institute, Santa Monica, Calif., USA
}

\section{Key Words}

Hypersensitivity reactions - Cisplatin - Carboplatin - Cancer chemotherapy

\begin{abstract}
The very early onset of platinum hypersensitivity reaction in a dentist treated with external beam radiation and weekly carboplatin for a locally advanced squamous cell carcinoma of the skin raises the provocative issue of whether occupational exposure to platinum may have contributed to this most unusual clinical event.
\end{abstract}

\section{Introduction}

The platinum agents are currently among the most widely employed anti-neoplastic drugs with demonstrated major biological and clinical activity in multiple tumor types.

While the individual agents (cisplatin, carboplatin, oxaliplatin) exhibit differing side effect profiles, they all share a most unfortunate risk for the development of hypersensitivity reactions. The signs and symptoms of these events can range from mild rash and diffuse erythroderma to serious anaphylactic reactions and death $[1,2]$.

One particularly highly characteristic feature of platinum allergic reactions is the observation that the initial clinical event (e.g. rash, dyspnea, hypotension) revealing hypersensitivity essentially always occurs following the delivery of multiple cycles (in most cases $>6$ ) of this specific class of drugs [1,2]. In fact, it is far more common for an allergic reaction to carboplatin or cisplatin in an ovarian cancer patient to be encountered during second-line administration of the agent (following 5-6 prior courses given in the frontline setting) than when one of the agents is infused as a component of front-line disease management [2].

It has been hypothesized that platinum hypersensitivity results from the stimulation of the immune system in susceptible individuals due to non-measurable concentrations of 


\begin{tabular}{c|l|l|l}
$\begin{array}{c}\text { Case Reports in } \\
\text { Oncology }\end{array}$ & $\begin{array}{l}\text { Case Rep Oncol 2010;3:294-297 } \\
\text { Dol: } 10.1159 / 000319835\end{array}$ & Published online: August 12, 2010 & $\begin{array}{l}\odot \text { 2010 S. Karger AG, Basel } \\
\text { ISSN 1662-6575 } \\
\text { www.karger.com/cro }\end{array}$ \\
\hline
\end{tabular}

metallic platinum contaminants (a known potent environmental allergen) during the drug manufacturing process [3-5]. However, since the actual quantity of systemic metallic platinum entering the body in this setting must be extremely small, repeated exposure is theorized to be necessary for the generation of an adequate immune response that will ultimately result in an allergic reaction.

A recently encountered patient with metastatic squamous cell carcinoma of the skin and no known prior exposure to a platinum chemotherapeutic agent was treated with weekly carboplatin plus external radiation, and developed a very surprising but unequivocal allergic drug reaction (diffuse rash) following only the second cycle of the chemotherapy. This case is briefly presented, along with a potential explanation for this highly unusual clinical course.

\section{Case Report}

A 78-year-old retired dentist was found to have squamous cell carcinoma of the skin with the primary site being the left upper chest. Unfortunately, a month after primary surgical resection he was found to have developed a $2.6 \mathrm{~cm}$ metastatic lesion in the lower neck (left side).

The mass was resected, but there was evidence of involvement in subcutaneous soft tissue and local lymph nodes. The tumor also extended to the deep soft tissue surgical margin.

The patient had a past medical history of bladder cancer, treated both surgically and with local instillations of BCG. No chemotherapy was administered. The patient had no known allergies.

Following discussions it was elected to treat the patient with external beam radiation to the neck along with weekly carboplatin (AUC 2), employed as a chemosensitization agent. The initial chemotherapy was given on day 1 of radiation, with the second cycle on day 8 .

The patient was noted to have a minimal diffuse rash on his legs prior to the administration of the third cycle of chemotherapy (day 15). A topical steroid was prescribed by a dermatologist.

One day after this treatment cycle the patient noted marked worsening of the rash which spread diffusely but spared the oral cavity. There was no fever or evidence of organ dysfunction. Oral steroids were administered and the rash gradually resolved. Radiation was continued without further delivery of platinum chemotherapy.

\section{Discussion}

As many as $10-15 \%$ of patients receiving platinum for $>6$ treatment cycles may be anticipated to experience an allergic event directly related to this class of anti-neoplastic agents [2]. The observation of a clinical platinum-associated hypersensitivity reaction following the administration of the initial several cycles of this agent is extremely uncommon. For example, in one report of more than 200 women with gynecologic malignancies who were routinely treated with a platinum drug in both the primary and second-line settings there were no cases of platinum allergy noted prior to the sixth cumulative cycle containing this class of drugs [2].

Why might the individual presented in this case report who was not previously exposed to platinum-based chemotherapy have developed a platinum allergy so soon following initiation of carboplatin?

Platinum hypersensitivity has been recognized for many years as being a serious occupational hazard for platinum miners [3-5]. While the current patient has no personal 
history of direct prior exposure to platinum, he was a dentist and platinum is a commonly employed component present in dental restorations $[6,7]$.

Is it possible that this individual who was clearly susceptible to the development of a platinum hypersensitivity reaction may have been exposed (perhaps over a period of many years) to very low concentrations of platinum during his work [8]? If this theory is correct then following the initial weekly carboplatin infusion there was stimulation of immune system resulting in a clinical event after the next (second) exposure to the agent.

It must be acknowledged that the preceding discussion is entirely speculative. However, it is consistent with both the historical facts and the clinical course of the allergic events in this patient.

Further, this case suggests that patients who experience what appears to be the surprisingly early onset of platinum hypersensitivity should be questioned regarding possible prior occupational exposure (e.g. jeweler, platinum miner, dentist) that may have been at least partially responsible for this unexpected outcome.

Nothing presented in this case report would argue that individual cancer patients with a known or suspected previous metallic platinum exposure should be denied the potential major clinical benefits associated with being given a platinum anti-neoplastic agent. However, knowledge of the patient's history (as in the current case) may be helpful in explaining most surprising and unusual clinical events. 


\section{References}

1 Zanotti KM, Markman M: Prevention and management of antineoplasticinduced hypersensitivity reactions. Drug Safety 2001;24:767-779.

2 Markman M, Kennedy A, Webster K, et al: Clinical features of hypersensitivity reactions to carboplatin. J Clin Oncol 1999;17:1141-1145.

-3 Cromwell O, Pepys J, Parish WE, et al: Specific IGE antibodies to platinum salts in sensitized workers. Clinical Allergy 1979;9:109-117.

4 Orbaek P: Allergy to the complex salts of platinum: A review of the literature and three case reports. Scand J Work Environ Health 1982;8:141-145.

5 Freedman SO, Krupey J: Respiratory allergy caused by platinum salts. J Allergy 1968;42:233-237.

6 Platinum Today. http://www.platinum.matthey.com/applications/industrialapplications/dental/ (accessed July 24, 2010).

7 Crown (dentistry). http://en.wikipedia.org/wiki/Crown_(dentistry) (accessed July 24, 2010).

-8 Hamann CP, Rodgers PA, Sullivan KM: Occupational allergens in dentistry. Curr Opin Allergy Clin Immunol 2004;4:403-409. 